\title{
Molecular and life-history effects of a natural toxin on herbivorous and non-target soil arthropods
}

\author{
A. E. Elaine van Ommen Kloeke • Cornelis A. M. van Gestel • \\ Bjarne Styrishave $\cdot$ Martin Hansen · Jacintha Ellers • \\ Dick Roelofs
}

Accepted: 13 January 2012/Published online: 5 February 2012

(C) The Author(s) 2012. This article is published with open access at Springerlink.com

\begin{abstract}
Natural toxins, such as isothiocyanate (ITC), are harmful secondary metabolites produced by plants. Many natural toxins occur in commercial crops, yet their possible negative repercussions on especially non-target soil organisms are largely unknown. This study examined life-history and gene transcriptional responses to 2-phenylethyl ITC on two soil arthropod species: Folsomia candida and Protaphorura fimata. To that end the standardized ISO guideline for ecotoxicological tests and a microarray for $F$. candida were used. The dissipation of 2-phenylethyl ITC in natural soil was investigated using GC-MS/MS for quantification. Half-lives, tested at four concentration levels in natural soil, were on average $16 \mathrm{~h}$ with biodegradation as the plausible main removal process. Regardless, toxic effects on reproduction were shown for $F$. candida and $P$. fimata, with EC50 values of around $11.5 \mathrm{nmol} / \mathrm{g}$ soil illustrating the toxic character of this compound. Gene expression profiles revealed the importance of fatty acid metabolism at low exposure concentrations (EC10), which is associated with the lipophilic nature of 2-phenylethyl ITC. At higher concentrations (EC50) gene expression
\end{abstract}

Electronic supplementary material The online version of this article (doi:10.1007/s10646-012-0861-z) contains supplementary material, which is available to authorized users.

A. E. E. van Ommen Kloeke $(\bowtie) \cdot$ C. A. M. van Gestel ·

J. Ellers · D. Roelofs

Department of Ecological Science, Faculty of Earth and Life

Sciences, VU University Amsterdam, De Boelelaan 1085,

$1081 \mathrm{HV}$ Amsterdam, The Netherlands

e-mail: elaine.van.ommenkloeke@vu.nl

B. Styrishave $\cdot$ M. Hansen

Department of Pharmaceutics and Analytical Chemistry, Faculty

of Pharmaceutical Sciences, University of Copenhagen,

Universitetsparken 2, 2100 Copenhagen, Denmark became more ubiquitous with over-expression of especially stress-related genes and sugar metabolism. The regulation of a gene encoding a precursor of follistatin, furthermore, implied the inhibition of reproduction and may be an important molecular target that can be linked to the observed adverse effect of life-history traits.

Keywords 2-Phenylethyl isothiocyanate · Soil dissipation - Ecotoxicology - Gene expression . Folsomia candida $\cdot$ Protaphorura fimata

\section{Introduction}

The soil ecosystem is extremely complex and comprises many different organisms, each with their own function, niche and interactions. Soil ecosystem functioning is challenged by many anthropogenic toxins such as metals (He et al. 2005), pesticides (Edwards and Bohlen 1992) and polycyclic aromatic hydrocarbons (Leon Paumen et al. 2009). These compounds may disrupt the soil ecosystem through mortality or reduced reproduction of soil organisms, as was shown by a large number of studies. Natural toxins, on the other hand, are rarely considered a threat to the environment yet can be lethal at low dosages. Natural toxins are organic compounds that are produced as secondary metabolites in fungi, bacteria, algae, plants or animals. Plant toxins (phytotoxins) are used by many edible plants as part of their natural defence system against pathogens and herbivory (Hoerger et al. 2009; van Egmond 2004).

Natural toxins can also be found in the plant family Brassicaceae, including broccoli, cabbage and mustard, which all produce glucosinolates (GSL) as secondary metabolites. Tissue damage, due to, for instance, herbivory, 
causes the enzyme myrosinase to hydrolyse GSL into several possible products of which the most common and most toxic is isothiocyanate (ITC) (Brown and Morra 1997; Halkier and Gershenzon 2006). ITCs have well-known negative effects on several invertebrate species such as fruit flies, wireworms, symphilids and nematodes (Brown and Morra 1997). For instance, $11 \mu \mathrm{M} 2$-phenylethyl ITC caused $50 \%$ mortality of populations of the root-knot nematode Meloidogyne incognita (Lazzeri et al. 2004). Toxic effects are mainly due to irreversible and nonspecific reactions of ITCs with proteins and amino acids, which result in inactivation of enzymes (Brown and Morra 1997).

Most ecological studies involving ITC have focussed on GSL-containing plants and their effects on specific plant pests such as insect herbivores (Hopkins et al. 2009). In contrast, little is known about effects on non-target species; beneficial or neutral species which are unintentionally affected by the toxicant via indirect contact (non-herbivory). Non-target soil organisms can be exposed to ITCs through the decomposition of crop litter, biofumigation or root exudates of GSL-containing plants (Wardle et al. 2004). ITCs do not seem to be target-specific (Brown and Morra 1997) and may, therefore, also affect non-target species. For instance, Jensen et al. (2010) showed a 50\% reduction in reproduction of the non-target soil arthropod, Folsomia fimetaria when exposed to $65 \mathrm{nmol}$ benzyl ITC per gram soil. Such effects on non-target, but beneficial soil organisms, can have severe negative repercussions on soil functioning.

The present study examined the effect of 2-phenylethyl ITC (the hydrolysis product of 2-phenylethyl GSL) on the survival and reproduction of the non-target fungivorous springtail Folsomia candida and the herbivorous springtail Protaphorura fimata. Springtails (Collembola) are one of the most abundant soil organisms, providing a significant contribution to nutrient mineralisation and decomposition (Berg et al. 2001; Filser 2002). Moreover, F. candida is an important model species for soil quality assessments (Fountain and Hopkin 2005). By complementing measurements on key life-history traits (survival and reproduction) with gene expression profiling (microarray techniques), we also aimed to assess the underlying modes of action of 2-phenylethyl ITC (van Straalen and Roelofs 2008). A microarray for $F$. candida was, therefore, applied to provide insight in the molecular response pathways invoked and the biological processes affected by 2-phenylethyl ITC. Concentrations of 2-phenylethyl ITC in the soil were measured over time, as ITCs are reported to be readily biodegradable (Brown and Morra 1997; Jensen et al. 2010). This is the first report on effects on soil organisms caused by a natural toxin in which measures of survival and reproduction are associated with molecular mechanistic information.

\section{Materials and methods}

Animals

Folsomia candida, a parthenogenetic collembolan (Fountain and Hopkin 2005), was used for both ecotoxicological and microarray experiments. A synchronized culture of either 10-12 days old (ecotoxicological experiments) or 23-25 days old (microarray) animals was prepared from the laboratory stock culture ("Berlin strain", VU University Amsterdam). This difference in age is chosen due to standardization and aims (reproduction and gene expression effects, respectively) of the existing protocols (ISO 1999; Nota et al. 2009) and the size of the animals at the time of harvesting; microarrays after 2 days and ecotoxicity tests after 28 days. Standardized methods for synchronization and stock maintenance followed the International Standardization Organization (ISO) guideline 11267 (ISO 1999). In short, springtails were kept on moistened plaster of Paris mixed with charcoal, at $20^{\circ} \mathrm{C}$, $75 \%$ relative humidity and a $12 \mathrm{~h}$ light/dark cycle. Protaphorura fimata, a herbivorous collembolan (Endlweber et al. 2009), also reproduces parthenogenetically and was cultured in a similar fashion as $F$. candida. This species is, however, difficult to synchronize (Heckmann et al. 2006). Therefore, a method was developed to synchronize animals with a maximum of 1 week difference in age. In short, a large quantity of 'almost ready to hatch' eggs (yellow/ brown coloured, oval shaped) were carefully transferred from the stock culture to a fresh Petri dish (with a bottom of moist plaster of Paris mixed with charcoal), using a dissecting needle. In order to keep the eggs clean (avoid microbial growth), $\sim 10$ adult females were included in this 'breeding dish'. After 1 week all juveniles were transferred to a fresh Petri dish using a glass Pasteur pipette. Each culture weekly received a fresh quantity of baker's yeast. To ensure that animals were reproductively active, they were only used for experiments if large quantities of eggs were present in the breeding dish. $P$. fimata used in the toxicity tests were 5-6 weeks old.

Experimental soil, compound and spiking

For all experiments natural LUFA 2.2 soil (Speyer, Germany) was used, which is a loamy sand soil with a $\mathrm{pH}$ of $( \pm \mathrm{SD}) \quad 5.5 \pm 0.1$ and an organic $\mathrm{C}$ content of $2.09 \pm 0.40 \%$. Before usage the soil was dried at $60^{\circ} \mathrm{C}$ for 24 h. 2-Phenylethyl ITC [2257-09-2] was obtained from Sigma Aldrich (www.sigmaaldrich.com) as a liquid solution (99\% purity). Ten percent of the total amount of soil needed for each treatment was spiked with the desired concentration of 2-phenylethyl ITC, ranging from 3.06 to $20.1 \mathrm{nmol}$ per g dry weight soil, by using stock solutions 
with acetone as solvent (1:1 ratio, i.e. $\mathrm{ml}$ acetone: $\mathrm{g}$ dry weight soil, DW) (Brinch et al. 2002). The spiked soils were thoroughly shaken and stored for $24 \mathrm{~h}$ in preservation jars to allow the soils to absorb the test chemical. Thereafter, to facilitate complete evaporation of all acetone, jars were left open overnight under a fume hood. Next, the remaining $90 \%$ of the total amount of soil needed for each treatment was mixed thoroughly in with the $10 \%$ spiked soil. Finally, the soil was moistened to $50 \%$ of the water holding capacity of $45.2 \%$, corresponding to $22 \%$ water of the soil DW.

\section{Measuring dissipation in soil}

To measure the soil dissipation rate of 2-phenylethyl ITC $5 \mathrm{~g}$ samples were taken from the soils spiked with 3.06-4.90-7.84-12.56-20.09 nmol/g soil, representing several concentrations within the test ranges used for the toxicity tests. Time intervals were: dry start (no water added to dry soil), wet start (hydrated soil, time 0), 1, 2, 3, 4, 24, 48, 120, $168 \mathrm{~h}$ after initial spiking. The samples were extracted by adding $5 \mathrm{ml}$ ethyl acetate and $100 \mu \mathrm{l}$ benzyl ITC $(500 \mu \mathrm{mol} / 1$ in ethyl acetate) as analytical internal standard (IS) to the samples which were then stored at $-18^{\circ} \mathrm{C}$ in darkness. Prior to analysis, samples were thawed, shaken on a vortex and the ethyl acetate phase was transferred on top of Pasteur pipettes packed with quartz wool inactivated, silica treated) in the bottom and $2.0 \mathrm{~g}$ anhydrous $\mathrm{Na}_{2} \mathrm{SO}_{4}$ above to filter and dry the samples. The purified sample eluate was collected in a $10 \mathrm{ml}$ test tube. This procedure was repeated with an additional $5 \mathrm{ml}$ of ethyl acetate added to the initial soil and again transferred to the same Pasteur pipette and eluate into the same test tube. The eluate was then evaporated with nitrogen to nearly dryness (less than $200 \mu \mathrm{l}$ ) and transferred to GC vials. Samples were analysed by gas chromatography tandem mass spectrometry (GC-MS/MS, Varian CP-3800 and Varian 1200 triple-quadruple) with electron ionization $(70 \mathrm{eV})$. The MS was operated in selected reaction ion monitoring mode acquiring data on the ion transitions $163.0>105.0$ for 2-phenylethyl ITC and $149.0>91.0$ for benzyl ITC. The GC-column was a $30 \mathrm{~m} \times 0.25 \mathrm{~mm}$, $0.25 \mu \mathrm{m}$ Factor Four VF-5 MS (Varian). The injection volume was $1 \mu \mathrm{l}$ in splitless mode achieved by a CTC CombiPAL autosampler and a PTV-injector kept at $200^{\circ} \mathrm{C}$ at all times. The initial column oven temperature was $40^{\circ} \mathrm{C}$ and the final temperature was $285^{\circ} \mathrm{C}\left(15^{\circ} \mathrm{C} / \mathrm{min}\right)$ with a total analysis time of $18.3 \mathrm{~min}$. Helium was used as carrier gas at $1.0 \mathrm{ml} / \mathrm{min}$. Calibration curves of both benzyl and 2-phenylethyl ITC were used to calculate the amount of 2-phenylethyl ITC present in the soil samples. First order degradation kinetics was used to estimate the degradation half-lives, which was performed in SPSS 15.0.
Ecotoxicological experiments

Ecotoxicological experiments to determine effects on the survival and reproduction for $F$. candida and $P$. fimata were carried out following the ISO guideline 11267 (ISO 1999). Concentrations of 2-phenylethyl ITC were for the F. candida experiment: 3.06-4.90-7.84-12.56-20.09 nmol/g soil and for the P. fimata experiment: 3.06-5.51-9.92$17.89-32.16 \mathrm{nmol} / \mathrm{g}$ soil. All experiments included a normal control (C) using only LUFA 2.2 soil and an acetone control (AC) with LUFA 2.2 soil spiked with only acetone. Samples were kept in $100 \mathrm{ml}$ jars and consisted of $30 \mathrm{~g}$ moist soil, ten animals, a few grains of baker's yeast and were incubated at $20^{\circ} \mathrm{C}, 70 \%$ relative humidity and a $12 \mathrm{~h}$ light/dark cycle. Once a week jars were aerated, moisture content was checked and fresh food added. After 28 days $100 \mathrm{ml}$ of water was added to test containers, stirred gently and completely poured out into a glass beaker. For each sample several digital photographs were taken to register all living springtails that came floating to the surface. To establish survival and total number of juveniles, photos were analyzed using the life science microscopy imaging software Cell ${ }^{\mathrm{D}}$ (Olympus) (Broerse and van Gestel 2010).

Dose-response curves were calculated to establish lethal concentrations (LC) and effect concentrations (EC, a specific percentage of reduction in juvenile production), using the logistic response model after (Haanstra et al. 1985):

$Y(c)=\frac{Y_{\max }}{1+\frac{x}{(100-x)}\left(\frac{c}{\mathrm{EC}_{x}}\right)^{b}}$

In which $Y$ is the percentage of survival $\left(\mathrm{LC}_{x}\right)$ or the number of juveniles for $\left(\mathrm{EC}_{x}\right)$, with $Y_{\max }$ the estimated maximum number in the untreated control, $x$ is the percentage of effect (here either 10 or 50), $c$ is the concentration and $b$ the slope of the dose-response curve. To investigate if the LC and EC values differed significantly between the two species a generalized likelihood ratio test was performed (Sokal and Rohlf 1995).

\section{Microarrays experiments}

Gene expression analysis was performed for $F$. candida exposed to four treatments including two toxic treatments and the water and acetone control. The two toxic treatments consisted of sub-lethal concentrations of 2-phenylethyl ITC close to EC10 and EC50 values for effects on reproduction $(6.21$ and $10.7 \mathrm{nmol} / \mathrm{g}$; being the average of the EC10 and EC50 values obtained in the present experiments and in an unpublished pilot experiment). Each treatment had four biological replicates, each consisting of 30 synchronized animals of 23-25 days old. After 2 days exposure, the flotation technique $(100 \mathrm{ml}$ demineralised 
water) was used to harvest animals from the soil. After scooping animals from the water surface with a spoon, they were placed on plaster of Paris to remove excess water. Quickly thereafter animals were placed in a micro-centrifuge tube and snap frozen in liquid nitrogen. RNA preparation, labelling and hybridisation were performed as described by Nota et al. (2009). For hybridisation a loop design was used (Fig. 1). Dyes were swapped at the biological replication level; for each treatment two samples were labelled with the fluorescent dye $\mathrm{Cy} 3$ and two with Cy5. A custom made 60 -mer, $8 \times 15 \mathrm{k}$ oligo microarray (Gene Expression Omnibus accession number: GPL7150) based on Agilent technologies was used for this experiment. This microarray contains 5,069 unique probes representing 5,069 different gene clusters from Collembase (www.collembase.org). All probes were spotted randomly in triplicate (Nota et al. 2009).

After feature extraction (Agilent FE software version 9.5.1.1) microarray data was further analysed using the package 'Limma' (version 2.18.3 Smyth (2004) in the statistical environment R (version 2.9.0). In short, normexp background correction (Ritchie et al. 2007), LOESS normalization (Smyth and Speed 2003) and aquantile normalization (Hahne et al. 2008) were performed for the whole dataset. As each microarray contained three replicates of the same probe, the consensus correlation, which is a robust average, was taken so that only one data point per microarray remained for each gene (Smyth et al. 2005). Each gene was thus represented by four data points (biological replicates/microarrays). Differential expression was then assessed by means of linear models and empirical

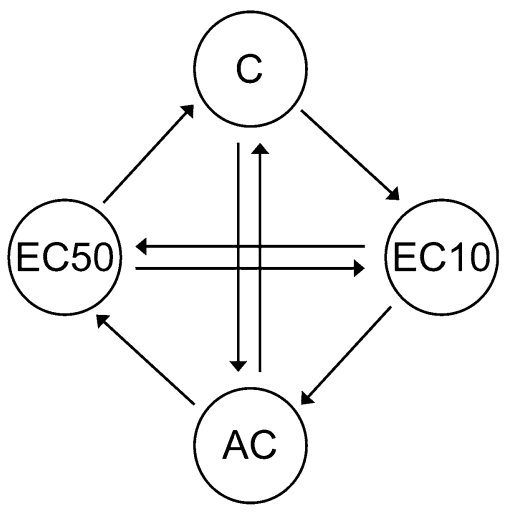

Fig. 1 Microarray hybridisation loop design for the four exposure treatments of Folsomia candida after $48 \mathrm{~h}$ exposure to 2-phenylethyl isothiocyanate in LUFA 2.2 soil. Each treatment had four biological replicates. $C$ control, $A C$ Aceton Control, EC10 concentration corresponding to a $10 \%$ reduction of reproduction and EC50 concentration corresponding to a $50 \%$ reduction of reproduction. Arrows indicate which samples were hybridized together on one twocoloured microarray. Arrow direction shows if a sample was labelled with CY3 (green) or CY5 (red): start of arrow $=\mathrm{CY} 3$ and end of arrow $=\mathrm{CY} 5$
Bayes methods. Finally the Benjamini-Hochberg's false discovery rate method (Benjamini and Hochberg 1995) was used for multiple testing corrections (adjusted $p<0.05$ was considered significant). MA-plots and boxplots were used for quality control of the data for each array. The expected and observed log ratios of the Agilent spike-in control probes showed a $R^{2}>0.95$ for all arrays. Differential expression analysis was performed for several contrasts between the four treatments resulting in a mean $\log 2$ expression ratio (treated/untreated) and a $p$-value for each probe on the array. Using Blast2GO (Conesa et al. 2005), part of the differentially expressed genes were annotated to known genes, assigned to a gene ontology (GO) term or referenced to an InterPro number, setting the hit threshold at e-value $<1$.0e- 6 . The raw and processed microarray data are available from the NCBI Gene Expression Omnibus (GEO) under accession number GSE29239.

\section{Results and discussion}

Dissipation of 2-phenylethyl ITC in natural soil

Recovery rates of 2-phenylethyl ITC varied between 52.8 and $78.6 \%$ based on the dry start concentrations. Adding water to the soil (dry vs. wet start) caused a significant decrease in the total concentration of 2-phenylethyl ITC in the soil $(p=0.03$, Student's $t$-test between measured concentrations of the dry and wet start). A similar effect of water addition was shown by Gimsing et al. (2009) for benzyl ITC, i.e. an increase in water content of the soil increased the degradation rate of benzyl ITC (Gimsing et al. 2009).

2-Phenylethyl ITC could only be detected in the LUFA soil samples for the first week (Fig. 2), indicating that this compound dissipates rapidly in natural soil. Half-lives were dependent on the starting concentrations and were 14.4

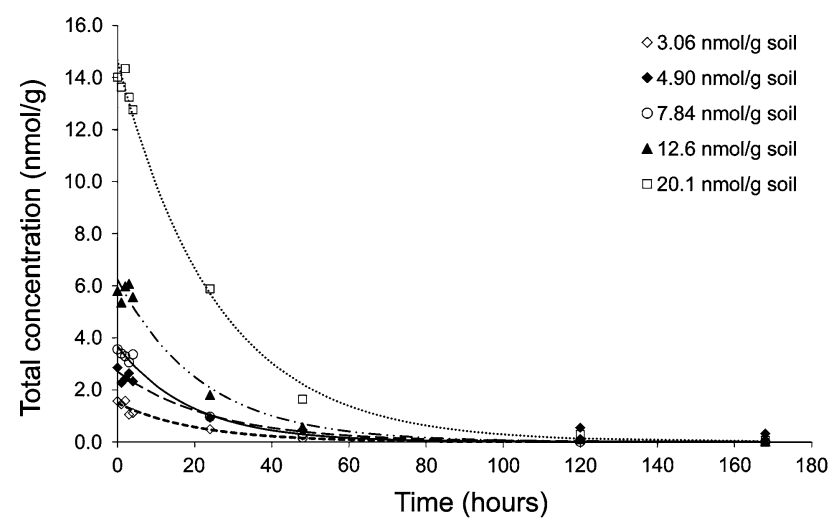

Fig. 2 Dissipation of 2-phenylethyl ITC (nmol/g soil) as a function of time (h) in moist LUFA 2.2 soil at $20^{\circ} \mathrm{C}$ for five different starting concentrations. Lines show fits of first-order degradation kinetics 
(95\% confidence interval: $6.50-22.04) \mathrm{h}$ for $3.06 \mathrm{nmol} / \mathrm{g}$ : $17.2(9.53-24.7) \mathrm{h}$ for $4.90 \mathrm{nmol} / \mathrm{g}, 13.1(9.67-16.6) \mathrm{h}$ for $7.84 \mathrm{nmol} / \mathrm{g}, 15.2(9.44-20.9) \mathrm{h}$ for $12.6 \mathrm{nmol} / \mathrm{g}$, and 17.6 (14.4-20.8) h for $20.1 \mathrm{nmol} / \mathrm{g}$ soil. For the $4.90 \mathrm{nmol} / \mathrm{g}$ data set the two last data points $(120$ and $168 \mathrm{~h})$ were excluded in the half-live model, as these values (still shown in Fig. 2), if included, would yield a very high and different half-life (55.9 h). Jensen et al. (2010) and Gimsing et al. (2009) showed similar dissipation patterns in nonsterile or natural soil for benzyl ITC, a compound with a chemical structure similar to 2-phenylethyl ITC. In sterile soils the dissipation of benzyl ITC was much slower, thus, indicating that microbial degradation is the chief driver responsible for the natural dissipation process of ITCs (Gimsing et al. 2009). We note that the stability of 2-phenylethyl ITC may also be influenced by the presence of test organisms and their associated food source, which was not investigated in this study.

Currently, data on ITC metabolites and their potential toxicity are unknown. Most likely 2-phenylethyl ITC is degraded to benzene, phenol and benzoic acid, which are deemed less toxic than the parent compound. However, as long as the toxicophore (NCS), the ITC part of the molecule, remains intact in any formed metabolite, the molecule is toxic. The potential effects of ITC metabolites, therefore, remain an important issue, which requires attention in future studies.

\section{Toxic effects on life-history traits}

Toxic effects of 2-phenylethyl ITC on reproduction of both Collembola species are presented in Fig. 3. On average, adult survival of $F$. candida was $( \pm \mathrm{SE}) 84 \pm 5.1 \%$ for the control (C) and $90 \pm 0.0 \%$ for acetone control (AC) group. Adult survival of $P$. fimata was on average $88 \pm 2.0 \%$ for

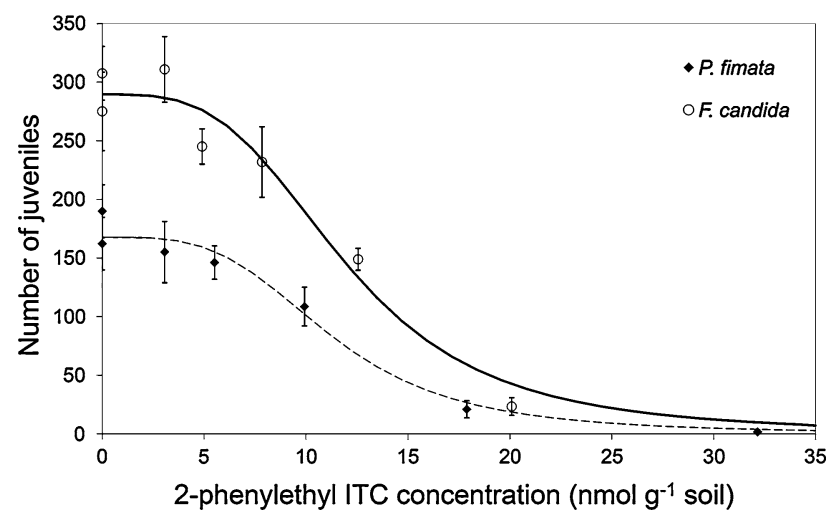

Fig. 3 Effects of 2-phenylethyl isothiocyanate (nominal concentrations) on the reproduction of Folsomia candida and Protaphorura fimata after 28 days exposure in LUFA 2.2 soil. Lines show the fit of the logistic dose response model to the data. Error bars are standard errors $(n=5)$
$\mathrm{C}$ and $90 \pm 3.2 \%$ for AC. On average $F$. candida produced $275 \pm 33.5$ juveniles in $\mathrm{C}$ and $307 \pm 22.9$ juveniles in $\mathrm{AC}$. For $P$. fimata the average number of juveniles was $162 \pm 22.5$ in $C$ and $190 \pm 22.6$ juveniles in AC. There was no significant difference in survival or reproduction between the two controls for both $F$. candida (survival: $p=0.273$ and reproduction: $p=0.448$, student's $t$-test) and $P$. fimata (survival: $p=0.608$ and reproduction: $p=0.412$, student's $t$-test). Although $P$. fimata is not a common test species for ecotoxicological tests, the species met the validity criteria described by the $F$. candida ISO guideline (1999) with regard to adult survival (mortality in controls $<20 \%$ ) and reproduction (per control vessel $>100$ juveniles).

2-Phenylethyl ITC proved to be toxic for both collembolan species. At exposure concentrations around $30 \mathrm{nmol} /$ g soil reproduction was completely impaired (Fig. 3). Estimated LC10, LC50, EC10 and EC50 values for both species (based on initial concentrations) are shown in Table 1. It should be noted that the LC and EC-values do not reflect the rapid loss of the compound from the soil. LC and EC values did not significantly differ between $F$. candida and $P$. fimata (Table 1), suggesting that 2-phenylethyl ITC exerts comparable effects on life-history traits of a non-target species as well as an herbivorous pest species. This supports the common assumption that ITCs are not target-specific (Brown and Morra 1997).

Furthermore, 2-phenylethyl ITC is approximately five times more toxic than benzyl ITC of which the EC50 is $65 \mathrm{nmol} / \mathrm{g}$ for $F$. fimetaria (Jensen et al. 2010). Apparently, a slight difference in chemical structure (one $\mathrm{CH}_{2}$ less) changes the toxicity of ITCs. Many studies show that 2-phenylethyl ITC is among the most toxic ITCs causing adverse effects on a wide range of soil organisms (van Dam et al. 2009). In comparison to anthropogenic toxicants this natural toxin is much more harmful. Fountain and Hopkin (2005) compiled a list of several metals and organic chemicals which were tested with $F$. candida using the ISO guideline (1999). Half of the listed organic chemicals have

Table 1 LC- and EC-values for the effects of 2-phenylethyl isothiocyanate on survival and reproduction, respectively of Folsomia candida and Protaphorura fimata after 28 days exposure in LUFA 2.2 soil. Values are in nmol ITC per gram soil (nominal concentrations)

\begin{tabular}{llllll}
\hline & F. candida & CI & P. fimata & CI & $\chi^{2}$ \\
\hline LC10 & 10.4 & $(8.67-12.1)$ & 9.32 & $(6.81-11.8)$ & 0.44 \\
LC50 & 15.4 & $(14.1-16.7)$ & 15.2 & $(13.5-17.0)$ & 0.03 \\
EC10 & 6.29 & $(3.73-8.84)$ & 6.08 & $(2.92-9.24)$ & 0.01 \\
EC50 & 12.0 & $(9.94-14.0)$ & 11.2 & $(8.63-13.8)$ & 0.18 \\
\hline
\end{tabular}

$C I=95 \%$ confidence interval. Life history trait differences between $F$. candida and $P$. fimata are deemed significant $(p<0.05)$ if $\chi^{2}>3.84$ 
higher EC50 values than 2-phenylethyl ITC (1.96 mg/ $\mathrm{kg}=12.0 \mathrm{nmol} / \mathrm{g}$ ) indicating lower toxicity (Fountain and Hopkin 2005).

The chemical structure of the compound gives insight as to why soil organisms can still come into contact with the toxin; the hydrophobic phenyl group binds firmly to the organic matter of the soil, while the ethyl tail (isothiocyanate), positioned outside soil particles, remains accessible to soil dwelling organisms (Potter et al. 1998). Furthermore, if bioavailable, lipophilic compounds easily bind to lipids, such as the hydrophobic tail region in phospholipid bilayer of cell membranes, which increases the permeability of membranes and contributes in turn to higher contact toxicity (van Dam et al. 2009).

Apparently, even though 2-phenylethyl ITC dissipates rapidly in natural soil and is only present in the soil for a few days, it is able to exert toxic effects on soil arthropods after 28 days. Adult death could be an explanation for reduction in reproduction. In that case the $\mathrm{LCx}$ and corresponding ECx should be overlapping. However, confidence intervals only slightly overlapped (Table 1). Reproduction thus also seems impaired in surviving adults on a physiological level, for which gene expression analysis can give more insight.

\section{Gene expression}

Differential expression in $F$. candida was analysed among 5,069 unique genes between control (C), acetone control (AC), versus EC10 and EC50. At both life-history levels, survival and reproduction, the two control groups $\mathrm{C}$ and AC did not significantly differ, even though animals in $\mathrm{AC}$ seemed to perform better. At the gene expression level, however, a total of 130 genes were significantly differentially expressed between $\mathrm{C}$ and $\mathrm{AC}$ (for a complete list we refer to Appendix A of the Supporting Information, Table A1). As 2-phenylethyl ITC was spiked into the soil with acetone as solvent, AC was considered the most appropriate control and thus used for further statistical analysis to identify significant transcriptional responses to EC10 and EC50 treatments.

The complete list of significantly expressed genes for the comparisons EC10 versus AC and EC50 versus AC can be found in the Supporting Information (Appendix A, Table A2). In the EC10 versus AC comparison, 75 genes were differentially expressed, of which 65 were up-regulated and 10 down-regulated (Table 2). In the EC50 versus AC comparison, 107 genes were differentially expressed, of which 64 were up-regulated and 43 down-regulated. Moreover, 46 genes showed similar differential expression at the EC10 and EC50 level (Fig. 4), so that 29 genes were differentially regulated only at the EC10 and 61 were unique to the EC50. Approximately $30 \%$ of all significant
Table 2 Total numbers of significantly differentially expressed genes in Folsomia candida exposed for $48 \mathrm{~h}$ to 2-phenylethyl ITC at concentrations similar to the 28-day EC10 and EC50 for effects on reproduction compared to the acetone control

\begin{tabular}{lll}
\hline & EC10 & EC50 \\
\hline Up & 65 & 64 \\
& 4994 & 4962 \\
Down & 10 & 43 \\
\hline
\end{tabular}

Non-differentially expressed genes are given in italics

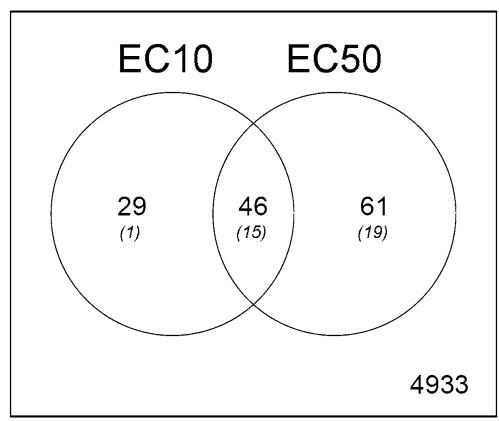

Fig. 4 Venn-diagram showing overlap in the total number of significantly differentially expressed genes in Folsomia candida after $48 \mathrm{~h}$ exposure to 2-phenylethyl isothiocyanate in LUFA 2.2 soil at concentrations corresponding to the EC10 and EC50 for effects on reproduction. Number of annotated genes are represented in brackets

genes were annotated using Blast2GO (Conesa et al. 2005). Thirty of the annotated and not-fully annotated differentially expressed genes were placed in the context of biological processes (Table 3) and are discussed in the following sections.

\section{Transcriptional response at EC10 level}

At the EC10 level, 2-phenylethyl ITC mainly induced metabolic processes (Table 3), especially lipid metabolism, which was indicated by the up-regulation of, for instance, acetyl-coenzyme A carboxylase alpha (Fcc00050), fatty acid delta-6 desaturase (Fcc01007), fatty acid synthase (Fcc02959) and genes encoding for the lipid transport proteins SEC14-like 1 and 2 (Fcc03602 and Fcc06054) (Saito et al. 2007). On the other hand, genes involved in lipid synthesis (diacylglycerol $o$-acyltransferase: Fcc03980) and the binding of small lipophilic molecules (cral-trio domain: Fcc04835), such as 2-phenylethyl ITC, were down-regulated. The cral-trio domain (sec-14 domain) also encompasses SEC 14-like proteins; this domain was thus represented in both the up-regulated and down-regulated genes. It is, however, likely that SEC14only proteins act as lipid transporters, while the multidomain SEC14-containing proteins (indicated by cral-trio) have more complex functions, integrating lipid metabolism with other biochemical processes (Saito et al. 2007). 
Table 3 Functional grouping of up- or down-regulated genes in Folsomia candida after $48 \mathrm{~h}$ exposure to 2-phenylethyl ITC in LUFA 2.2 soil at concentrations corresponding to the EC10 (6.21 nmol/g soil) and/or EC50 (10.7 nmol/g soil) for effects on reproduction compared to the acetone control

\begin{tabular}{|c|c|c|c|c|c|c|c|}
\hline Process & Gene name & Blast2GO description & E-value & E-code & InterPro & $\begin{array}{l}\mathrm{EC} 10 \\
\log \mathrm{FC}\end{array}$ & $\begin{array}{l}\text { EC50 } \\
\log F C\end{array}$ \\
\hline \multicolumn{8}{|c|}{ Fatty acid metabolism } \\
\hline GO:0006633 & Fcc00050 a & Acetyl-coenzyme a carboxylase alpha & $3.32 \mathrm{E}-82$ & EC:6.4.1.2 & & 0.961 & 1.086 \\
\hline GO:0006629 & Fcc01007 & Delta-5 fatty acid desaturase & $7.10 \mathrm{E}-34$ & & IPR005804 & 0.989 & \\
\hline GO:0006633 & Fcc02959 ${ }^{\mathrm{a}}$ & Fatty acid synthase & 4.94E-23 & EC:1.3.1.10 & IPR000794 & 1.082 & 1.266 \\
\hline GO:0080090 & Fcc00142 & Sterol regulatory element-binding protein 1 & $1.43 \mathrm{E}-15$ & & & & 0.458 \\
\hline \multirow[t]{5}{*}{ GO:0045540 } & Fcc06054 & Sec14-like 2 (cerevisiae) & $9.79 \mathrm{E}-09$ & & IPR001251 & 0.693 & 0.594 \\
\hline & Fcc03602 & Sec14-like 1 (cerevisiae) & $1.85 \mathrm{E}-15$ & & IPR001251 & 0.681 & \\
\hline & Fcc04835 & $\begin{array}{l}\text { Sec14-like protein } 4 \text { (cral trio } \\
\text { domain-containing protein) }\end{array}$ & $1.44 \mathrm{E}-17$ & & IPR001251 & -1.184 & \\
\hline & Fcc03980 & Diacylglycerol o-acyltransferase & $2.44 \mathrm{E}-09$ & & & -1.554 & \\
\hline & Fcc03847 & Alkaline ceramidase & $2.66 \mathrm{E}-16$ & & & 0.643 & \\
\hline \multicolumn{8}{|l|}{ Oxidative stress } \\
\hline GO:0005515 & Fcc00494 ${ }^{\mathrm{a}}$ & Glutathione s-transferase & 7.64E-39 & & IPR004045 & 0.703 & 0.830 \\
\hline GO:0005515 & Fcc05973 ${ }^{\mathrm{a}}$ & Glutathione s-transferase & $1.48 \mathrm{E}-42$ & & IPR004045 & & 1.123 \\
\hline GO:0055114 & Fcc02155 ${ }^{\mathrm{a}}$ & Cytochrome p450 & $2.84 \mathrm{E}-32$ & & IPR001128 & & 1.002 \\
\hline GO:0005215 & Fcc04663 ${ }^{\mathrm{a}}$ & Alpha-tocopherol transfer & $4.36 \mathrm{E}-42$ & & IPR001071 & & -0.746 \\
\hline GO:0006979 & Fcc00022 $2^{\mathrm{a}}$ & $\operatorname{Rec} 8$ & $1.54 \mathrm{E}-20$ & & IPR002007 & & -1.150 \\
\hline \multicolumn{8}{|c|}{ Immune response } \\
\hline GO:0009058 & Fcc00057 & Isopenicillin $\mathrm{n}$ synthetase & $2.16 \mathrm{E}-29$ & & & & 0.856 \\
\hline GO:0006950 & Fcc03623 ${ }^{\mathrm{a}}$ & Advillin & $2.71 \mathrm{E}-47$ & & IPR003128 & 0.604 & 0.596 \\
\hline \multicolumn{8}{|c|}{ Sugar metabolism } \\
\hline GO:0003824 & Fcc05611 & Trehalase & $2.51 \mathrm{E}-46$ & & IPR001661 & & 0.868 \\
\hline GO:0050660 & Fcc04789 ${ }^{\mathrm{a}}$ & Glucose dehydrogenase & $1.54 \mathrm{E}-15$ & & IPR007867 & & 0.827 \\
\hline GO:0006565 & Fcc02499 ${ }^{a}$ & Cystathionine beta-synthase & $5.25 \mathrm{E}-61$ & EC:4.2.1.22 & & & 0.788 \\
\hline GO:0009744 & Fcc00529a & Maltase- intestinal-like & $8.26 \mathrm{E}-30$ & EC:3.2.1.26 & IPR000322 & 1.188 & 1.309 \\
\hline GO:0015986 & Fcc03280 ${ }^{a}$ & Vacuolar atpase subunit $d$ & $5.11 \mathrm{E}-71$ & EC:3.6.3.6 & IPR002699 & & -0.582 \\
\hline \multicolumn{8}{|c|}{ Growth and development } \\
\hline GO:0005214 & Fcc03188 & Adult cuticle & $3.21 \mathrm{E}-17$ & & IPR000618 & & -0.801 \\
\hline \multirow[t]{2}{*}{ GO:0005576 } & Fcc04617 & Follistatin precursor & $6.66 \mathrm{E}-29$ & & IPR001239 & & -1.351 \\
\hline & Fcc00558 & Chitin binding peritrophin- & 4.14E-07 & & & 0.595 & \\
\hline GO:0006032 & Fcc02522 ${ }^{\mathrm{a}}$ & Endochitinase & $1.33 \mathrm{E}-29$ & & IPR000726 & 1.110 & 1.080 \\
\hline GO:0006032 & Fcc00881 ${ }^{\mathrm{a}}$ & Acidic mammalian chitinase-like & $1.40 \mathrm{E}-51$ & EC:3.2.1.0 & IPR001223 & 1.124 & 0.889 \\
\hline GO:0008152 & Fcc $03673^{\mathrm{a}}$ & Juvenile hormone acid methyltransferase & $2.34 \mathrm{E}-23$ & EC:2.1.1.0 & IPR013216 & 0.794 & 1.034 \\
\hline \multicolumn{8}{|l|}{ Transcription } \\
\hline GO:0043967 & Fcc03584 ${ }^{\mathrm{a}}$ & Dna methyltransferase 1 associated protein 1 & $9.69 \mathrm{E}-71$ & & & & -0.518 \\
\hline GO:0003899 & Fcc04065 ${ }^{\mathrm{a}}$ & Dna-directed rna polymerase ii subunit rpb4 & $1.61 \mathrm{E}-51$ & $\mathrm{EC}: 2.7 .7 .6$ & IPR005574 & & -0.716 \\
\hline
\end{tabular}

Bold indicate up-regulation of genes while italic indicate down-regulation of genes. Empty cells indicate the gene was not differentially expressed compared to the acetone control

Process Biological process category and related GO-code of gene (if available), GeneName Collembase accession number, Blast Description Best Blast2GO hit, E-value Blast2GO E-value, E-Code Enzyme Commission number, InterPro first code of hits found in InterPro database, $\log F C$ the $\log 2$ transformed ratio (treated/untreated)

a Sequence was fully annotaded by Blast2GO

b Second best annotated Blast2GO description for this sequence

Alkaline ceramidase (Fcc03847) was also up-regulated, which codes for an enzyme that is part of a complex bioactive lipid system that mediates cell proliferation, differentiation, apoptosis, adhesion, and migration. It hydrolyses ceramide to sphingosine, a mediator of cellgrowth arrest and apoptosis (Mao and Obeid 2008). 
Moreover, Fcc00558 was up-regulated. Although not fully annotated by Blast2GO, this sequence had high similarity with a gene encoding for chitin binding peritrophin, which functions in the formation of the extracellular envelope from chitin (peritrophic matrix). This envelope protects the mid-gut of arthropods from physical damage by food particles and from attacks of plant toxins such as 2-phenylethyl ITC (Merzendorfer and Zimoch 2003). Activity of such a process was supported by the up-regulation of genes involved with chitinase (endochitinase: Fcc02522 and acidic mammalian chitinase-like: Fcc00881), an enzyme involved in the moulting process. Taken together, gene expression at the EC10 level revolved around fatty acid metabolism and moulting processes, which can be associated with the lipophilic nature of 2-phenylethyl ITC.

\section{Transcriptional response at EC50 level}

The effects of 2-phenylethyl ITC on gene expression at the EC50 level were more diverse than the EC10 response (Table 3). Up-regulation of a single cytochrome P450 gene (CYP450: Fcc02155) and two glutathione s-transferases (GST: Fcc00494 and Fcc05973) indicated detoxification by phase I and II biotransformation of 2-phenylethyl ITC to be processed for elimination through the aqueous phase. It is well known that genes coding for CYP450 and GST contribute to the biotransformation of xenobiotics and are oxidative stress responsive as described by their associated GO term (Hayes and Pulford 1995). Interestingly, this process is also activated in humans exposed to 2-phenylethyl ITC and is supposed to be associated with chemoprevention (Cheung and Kong 2010), suggesting that 2-phenylethyl ITC is a xenobiotic compound. Genes associated with vitamin $\mathrm{E}$ transport (alpha-tocopherol transfer: Fcc004663), known for its anti-oxidant properties (Dutta-Roy 1999), and oxidation reduction (rec8: Fcc00022) were, however, down-regulated. In addition, isopenicillin $\mathrm{N}$ synthase (Fcc00057) and advillin (Fcc03623) were up-regulated. These genes are involved in microbial defence and immune response, suggesting a more general stress response.

Furthermore, 2-phenylethyl ITC affects transcription of genes involved in sugar metabolism in $F$. candida. This is illustrated by the up-regulation of, for instance, trehalase (Fcc05611), glucose dehydrogenase (Fcc04789), cystathionine beta-synthase (Fcc02499) and maltase-intestinal-like (Fcc00529). These transcriptional responses probably serve to increase energy production, which was supported by the down-regulation of vacuolar ATPase subunit d (Fcc03280), an enzyme that converts ATP into ADP. Up-regulation of trehalase was also found as a response of $F$. candida to desiccation stress (Timmermans et al. 2009). Changes in sugar metabolism indicate that organisms invest in many energyconsuming processes, in order to survive stressful conditions.
In addition, a gene encoding the formation of adult cuticle (Fcc03188), important for development and growth, was exclusively down-regulated at the EC50 level. For sequence Fcc04617, the second best Blast2GO hit with a similarity of $79 \%$, coded for a precursor of follistatin. Genes involved with follistatin have been shown to modulate activity of members of the TGF- $\beta$ super family (bone morphogenic proteins 2 and 4) in vertebrates, which in turn are important for e.g. axis formation, development of the nerve system and embryonic development. In Drosophila, follistatin is expressed throughout development where it is, for instance, important for morphogenesis in pupae (Bickel et al. 2008; Pentek et al. 2009). Perturbation of this gene may, therefore, result in failure of early embryonic development and as such overall reduction in reproduction. However, care should be taken to directly extrapolate gene expression to life history effects due to the differences in exposure conditions between ecotoxicological and microarray experiments.

Finally, other gene expression patterns involved the down-regulation of DNA methyltransferase (Fcc03584), mediating DNA methylation, and DNA-directed RNA polymerase ii subunit rpb4 (Fcc04065), involved in RNA synthesis. This suggests that specific parts of DNA, e.g. stress response genes, were more accessible while overall mRNA production was decreased.

\section{Overlap in genes between EC10 and EC50}

A large overlap was found for gene expression at the EC10 and EC50 treatments (Table 3), which also showed similar direction of regulation (up- or down) for both treatments. Three particular genes, up-regulated in both treatments, are worth mentioning. The first two are genes encoding for endochitinase (Fcc02522) and acidic mammalian chitinase (Fcc00881). Chitinase is an enzyme which is a key constituent of the moulting process of the cuticle and the peritrophic matrix of arthropods (Merzendorfer and Zimoch 2003). Apparently, the moulting process was stimulated as a reaction to the toxin. We speculate that this points towards the lipophilic nature of 2-phenylethyl ITC, resulting in stimulated excretion of epithelium to which the compound is bound. Second, a gene coding for juvenile hormone acid methyltransferase (Fcc03673) was up-regulated in both treatments. Juvenile hormone acid methyltransferase is an enzyme involved in the final step of juvenile hormone synthesis. This group of acyclic sesquiterpenoids regulate many physiological processes such as development, growth, reproduction and diapause (Shinoda and Itoyama 2003). Up-regulation of this gene indicates that these crucial life-cycle processes of $F$. candida are affected by 2-phenylethyl ITC. 


\section{Conclusion}

2-Phenylethyl ITC had a noticeable effect on survival and reproduction of both springtail species and the gene expression of $F$. candida. These gene profiles represent hypotheses for explaining the underlying modes of action and acute effects of the toxin on survival and reproduction. For instance, due to the lipophilic nature of the ITC, we observed genes involved in fatty acid metabolism to be severely affected and overall gene activity to be altered. This in turn activated genes encoding biotransformation enzymes, to counteract toxic activity and facilitate excretion of cyclic compounds such as ITC. The regulation of a gene encoding follistatin, furthermore, implied the inhibition of reproduction and may be an important molecular target that can be linked to the observed adverse effect of life history traits. This study clearly shows the detrimental effect of this natural toxin on beneficial non-target and herbivorous springtails. Loss of especially beneficial, functionally important soil invertebrates can result in serious negative repercussions on soil ecosystem functioning (Berg et al. 2001). Natural toxins are present in many agricultural systems and probably at increased levels in the near future. Current interests in ITCs focus on the toxic characteristics that have, for instance, been exploited for alternative pest management methods, socalled biofumigation (Matthiessen and Kirkegaard 2006). Several field and laboratory studies have shown that ITC concentrations can rise up to $100 \mathrm{nmol} / \mathrm{g}$ soil after using effective biofumigation strategies (Gimsing and Kirkegaard 2009). Other interests concern the possible chemopreventive nature of ITCs involved with cancer (Traka and Mithen 2009). Such social-economic uses of ITC call for novel commercial health crop varieties with enhanced GSL levels (Traka and Mithen 2009). By definition, as indicated by their secondary metabolite nature, they exert toxicological effects (Hoerger et al. 2009). Potential risks to the environment of these natural toxins, readily available in every-day crops, should therefore be carefully assessed. This study is the first step towards an understanding of these potential risks by studying the effects of a natural toxin at a life-history trait and molecular level.

Acknowledgments This project is funded by the research program Ecology Regarding Genetically modified Organisms (ERGO) of the Netherlands Organization of Scientific Research (NWO, project no.: 838.06.091). Individuals to start our own Protaphorura fimata stock were kindly provided by Lars-Henrik Heckman from the National Environmental Research Institute, Silkeborg, Denmark. We would like to thank the microarray facility of the VUmc, Amsterdam for their laboratory assistance and Thierry Janssens and Benjamin Nota (VU University, Amsterdam) for advice on microarray analysis.
Open Access This article is distributed under the terms of the Creative Commons Attribution License which permits any use, distribution, and reproduction in any medium, provided the original author(s) and the source are credited.

\section{References}

Benjamini Y, Hochberg Y (1995) Controlling the false discovery rate: a practical and powerful approach to multiple testing. J R Stat Soc Ser BMethodol 57:289-300

Berg M, de Ruiter P, Didden W, Janssen M, Schouten T, Verhoef H (2001) Community food web, decomposition and nitrogen mineralisation in a stratified Scots pine forest soil. Oikos 94:130-142

Bickel D, Shah R, Gesualdi SC, Haerry TE (2008) Drosophila follistatin exhibits unique structural modifications and interacts with several TGF-beta family members. Mech Dev 125:117-129

Brinch UC, Ekelund F, Jacobsen CS (2002) Method for spiking soil samples with organic compounds. Appl Environ Microbiol 68: $1808-1816$

Broerse M, van Gestel CAM (2010) Mixture effects of nickel and chlorpyrifos on Folsomia candida (Collembola) explained from development of toxicity in time. Chemosphere 79:953-957

Brown PD, Morra MJ (1997) Control of soil-borne plant pests using glucosinolate-containing plants. Adv Agron 61:167-231

Cheung KL, Kong AN (2010) Molecular targets of dietary phenethyl isothiocyanate and sulforaphane for cancer chemoprevention. Am Assoc Pharm Sci J 12:87-97

Conesa A, Gotz S, Garcia-Gomez JM, Terol J, Talon M, Robles M (2005) Blast2GO: a universal tool for annotation, visualization and analysis in functional genomics research. Bioinformatics 21:3674-3676

Dutta-Roy AK (1999) Molecular mechanism of cellular uptake and intracellular translocation of alpha-tocopherol: role of tocopherol-binding proteins. Food Chem Toxicol 37:967-971

Edwards CA, Bohlen PJ (1992) The effects of toxic chemicals on earthworms. Rev Environ Contam Toxicol 125:23-99

Endlweber K, Ruess L, Scheu S (2009) Collembola switch diet in presence of plant roots thereby functioning as herbivores. Soil Biol Biochem 41:1151-1154

Filser J (2002) The role of Collembola in carbon and nitrogen cycling in soil. Pedobiologia 46:234-245

Fountain MT, Hopkin SP (2005) Folsomia candida (Collembola): a "standard" soil arthropod. Ann Rev Entomol 50:201-222

Gimsing AL, Kirkegaard JA (2009) Glucosinolates and biofumigation: fate of glucosinolates and their hydrolysis products in soil. Phytochem Rev 8:299-310

Gimsing AL, Strobel BW, Hansen HCB (2009) Degradation and sorption of 2-propenyl and benzyl isothiocyanate in soil. Environ Toxicol Chem 28:1178-1184

Haanstra L, Doelman P, Voshaar JHO (1985) The use of sigmoidal dose-response curves in soil ecotoxicological research. Plant Soil 84:293-297

Hahne F, Huber W, Gentleman R, Falcon S (2008) Bioconductor case studies. Springer, New York

Halkier BA, Gershenzon J (2006) Biology and biochemistry of glucosinolates. Ann Rev Plant Biol 57:303-333

Hayes JD, Pulford DJ (1995) The glutathione S-Transferase supergene family: regulation of GST and the contribution of the isoenzymes to cancer chemoprotection and drug resistance. Crit Rev Biochem Mol Biol 30:445-600 
He ZLL, Yang XE, Stoffella PJ (2005) Trace elements in agroecosystems and impacts on the environment. J Trace Elem Med Biol 19:125-140

Heckmann LH, Griffiths BS, Caul S, Thompson J, Pusztai-Carey M, Moar WJ, Andersen MN, Krogh PH (2006) Consequences for Protaphorura armata (Collembola: Onychiuridae) following exposure to genetically modified Bacillus thuringiensis (Bt) maize and non-Bt maize. Environ Pollut 142:212-216

Hoerger CC, Schenzel J, Strobel BW, Bucheli TD (2009) Analysis of selected phytotoxins and mycotoxins in environmental samples. Anal Bioanal Chem 395:1261-1289

Hopkins RJ, van Dam NM, van Loon JJA (2009) Role of glucosinolates in insect-plant relationships and multitrophic interactions. Ann Rev Entomol 54:57-83

ISO (1999) Soil quality: inhibition of reproduction of Collembola (Folsomia candida) by soil pollutants. ISO 11267. International Standardization Organization, Geneva

Jensen J, Styrishave B, Gimsing AL, Hansen HCB (2010) The toxic effects of benzyl glucosinolate and its hydrolysis product, the biofumigant benzyl isothiocyanate, to Folsomia fimetaria. Environ Toxicol Chem 29:359-364

Lazzeri L, Curto G, Leoni O, Dallavalle E (2004) Effects of glucosinolates and their enzymatic hydrolysis products via myrosinase on the root-knot nematode Meloidogyne incognita (Kofoid et White) Chitw. J Agric Food Chem 52:6703-6707

Leon Paumen M, de Voogt P, van Gestel CAM, Kraak MHS (2009) Comparative chronic toxicity of homo- and heterocyclic aromatic compounds to benthic and terrestrial invertebrates: generalizations and exceptions. Sci Total Environ 407:4605-4609

Mao CG, Obeid LM (2008) Ceramidases: regulators of cellular responses mediated by ceramide, sphingosine, and sphingosine1-phosphate. Biochimica Et Biophysica Acta-Mol Cell Biol Lipids 1781:424-434

Matthiessen JN, Kirkegaard JA (2006) Biofumigation and enhanced biodegradation: opportunity and challenge in soilborne pest and disease management. Crit Rev Plant Sci 25:235-265

Merzendorfer H, Zimoch L (2003) Chitin metabolism in insects: structure, function and regulation of chitin synthases and chitinases. J Exp Biol 206:4393-4412

Nota B, Bosse M, Ylstra B, van Straalen NM, Roelofs D (2009) Transcriptomics reveals extensive inducible biotransformation in the soil-dwelling invertebrate Folsomia candida exposed to phenanthrene. BMC Genomics 10:236

Pentek J, Parker L, Wu A, Arora K (2009) Follistatin preferentially antagonizes activin rather than BMP signaling in Drosophila. Genesis 47:261-273
Potter MJ, Davies K, Rathjen AJ (1998) Suppressive impact of glucosinolates in Brassica vegetative tissues on root lesion nematode Pratylenchus neglectus. J Chem Ecol 24:67-80

Ritchie ME, Silver J, Oshlack A, Holmes M, Diyagama D, Holloway A, Smyth GK (2007) A comparison of background correction methods for two-colour microarrays. Bioinformatics 23:2700 2707

Saito K, Tautz L, Mustelin T (2007) The lipid-binding SEC 14 domain. Biochimica Et Biophysica Acta-Mol Cell Biol Lipids 1771:719-726

Shinoda T, Itoyama K (2003) Juvenile hormone acid methyltransferase: a key regulatory enzyme for insect metamorphosis. Proc Natl Acad Sci USA 100:11986-11991

Smyth GK (2004) Linear models and empirical bayes methods for assessing differential expression in microarray experiments. Stat Appl Genet Mol Biol 3:Article3

Smyth GK, Speed T (2003) Normalization of cDNA microarray data. Methods 31:265-273

Smyth GK, Michaud J, Scott HS (2005) Use of within-array replicate spots for assessing differential expression in microarray experiments. Bioinformatics 21:2067-2075

Sokal RR, Rohlf FJ (1995) Biometry the principles and practise of statistics in biological research. W.H. Freeman and company, New York

Timmermans M, Roelofs D, Nota B, Ylstra B, Holmstrup M (2009) Sugar sweet springtails: on the transcriptional response of Folsomia candida (Collembola) to desiccation stress. Insect Mol Biol 18:737-746

Traka M, Mithen R (2009) Glucosinolates, isothiocyanates and human health. Phytochem Rev 8:269-282

van Dam NM, Tytgat TOG, Kirkegaard JA (2009) Root and shoot glucosinolates: a comparison of their diversity, function and interactions in natural and managed ecosystems. Phytochem Rev 8:171-186

van Egmond HP (2004) Natural toxins: risks, regulations and the analytical situation in Europe. Anal Bioanal Chem 378:1152-1160

van Straalen NM, Roelofs D (2008) Genomics technology for assessing soil pollution. J Biol 7:19

Wardle DA, Bardgett RD, Klironomos JN, Setälä H, van der Putten WH, Wall DH (2004) Ecological linkages between aboveground and belowground biota. Science 304:1629-1633 